\title{
New Package Scheme of a 2.5-Gb/s Plastic Transceiver Module Employing Multiwall Nanotubes for Low Electromagnetic Interference
}

\author{
Chia-Ming Chang, Jin-Chen Chiu, Wern-Shiang Jou, Tzong-Lin Wu, Senior Member, IEEE, \\ and Wood-Hi Cheng, Senior Member, IEEE
}

\begin{abstract}
A novel polymer-based multiwall carbon nanotube (MWCNT) with high shielding effectiveness (SE) for use in packaging a $2.5-\mathrm{Gb} / \mathrm{s}$ plastic transceiver module is demonstrated. The MWCNT composites are tested to evaluate the electromagnetic (EM) shielding against emitted radiation from the optical transceiver modules. The results show that the SE of MWCNT composite packages exhibit $\mathbf{3 8}-\mathbf{4 5} \mathrm{dB}$ in the far-field source and $28-40 \mathrm{~dB}$ in the near-field source at a frequency range of $1-3 \mathrm{GHz}$, and an average of $14 \mathrm{~dB}$ for the optical transceiver modules at a frequency of $2.5 \mathrm{GHz}$. The MWCNT composites with their high SE are potentially suitable for packaging low-cost and low-EMinterference optical transceiver modules used in Gigabit Ethernet or fiber-to-the-home lightwave transmission systems.
\end{abstract}

Index Terms-Electromagnetic interference (EMI), multiwall carbon nanotubes (MWCNTs), plastic optical transceiver modules, shielding effectiveness.

\section{INTRODUCTION}

$\mathbf{T}$ HE widespread deployment of low-cost optical access networks for fiber-to-the-home (FTTH) applications will necessitate a considerable reduction in the cost of key components such as optical transceiver modules. Optical transceiver module costs are primarily dependent on their packaging. Owing to its low-cost nature and ease of manufacture, the plastic packaging technology has been considered as one of the major choices for reducing the costs of fabricating optical transceiver modules for use in the FTTH applications [1]-[5].

However, plastics alone are inherently transparent to electromagnetic (EM) radiation, and hence providing no shielding against radiation emissions. To improve the EM shielding for the plastic packaging, electronic conductive properties have to be added into the plastic hosts for adequate EM shielding. The currently available techniques for preventing EM interference

Manuscript received January 9, 2005; revised May 16, 2006. This work was supported in part by the MOP Program for Promoting Academic Excellence of University, R.O.C., under Grant A-91-E-FA08-1-4.

C.-M. Chang is with Philips Semiconductor Kaohsiung, Kaohsiung 811, Taiwan, R.O.C., and also with the Institute of Electro-Optical Engineering, National Sun Yat-sen University, Kaohsiung 804, Taiwan, R.O.C.

J.-C. Chiu and W.-H. Cheng are with the Institute of Electro-Optical Engineering, National Sun Yat-sen University, Kaohsiung 804, Taiwan, R.O.C. (e-mail: whcheng@mail.nsysu.edu.tw).

W.-S. Jou is with the Department of Mold and Die Engineering, National Kaohsiung University of Applied Science, Kaohsiung 807, Taiwan, R.O.C.

T.-L. Wu is with the Department of Electrical Engineering, National Taiwan University, Taipei 106, Taiwan, R.O.C.

Digital Object Identifier 10.1109/JSTQE.2006.879534
(EMI) shielding include conductive sprays, conductive fillers, zinc-arc spraying, electroplating or electrolysis plating on housing surfaces, modifications of electrical properties during the molding stage, and other metallization processes. Among these methods, the most popular one for EM shielding is to compound plastics with discontinuous electronic conductive fillers such as metal particles, metal flakes, stainless fiber, graphitized carbon particles, graphitized carbon fibers, metal-coated glass, and carbon fibers [6], [7].

Experimental evidence has shown that the carbon fibers with an aspect ratio of 1000 are good conductive fillers for providing a high EM shielding [8], [9]. The optical transceiver modules employing nylon and liquid crystal polymer (LCP) reinforced with carbon fiber showed that the measured shielding effectiveness (SE) was over $20 \mathrm{~dB}$ with $25 \%$ of weight percentage of the carbon fiber mixed in the nylon and LCP composites [3], [4]. In addition, the SE performance will be higher for higher weight percentages of the carbon fiber and thicker material of the carbon-fiber mixture. However, with such plastic composites, the dominant cost of the package is the carbonfiber fillers. Therefore, developing a plastic composite housing with a low weight percentage of carbon fiber and with good shielding ability is necessary for fabricating a low cost and high SE optical transceiver module.

Recently, low cost, lightweight, and high EM shielding package for the optical transceiver module has been made by employing the woven continuous carbon-fiber (WCCF) epoxy composite with the compression molding technology [10]. Epoxy resins are one of the best matrix materials for carbon-fiber composites because they adhere well to a wide variety of carbon fibers. By weaving the continuous carbon fiber in a balanced twill structure (BTS) with excellent conductive networks, we found that the SE of the package housing, while keeping a very low weight percentage $(4.8 \%)$ of carbon fiber, can reach about $80 \mathrm{~dB}$ under the far-field source measurement and about $25 \mathrm{~dB}$ in the near-field source measurement [10].

As the electronic and mechanical properties of carbon nanotubles (CNTs) are remarkable [11], [12], the CNTs have been the focus of considerable research and development for use in nanoscale electronic and optoelectronic applications such as integrated circuit (IC) interconnections [13] and optical emission devices [14]. CNTs may be considered as one of the electronic conductive fillers for EM shielding of plastic-package applications because of their smaller diameter, higher aspect ratios, higher conductivity, and better mechanical properties. The 
aspect ratios of most CNTs could be higher than 1000, which offers a good condition to form overlapping conductive CNTs networking to provide a high EM shielding.

In this paper, a novel polymer-based multiwall carbon nanotube (MWCNT) with high SE for use in packaging a 2.5$\mathrm{Gb} / \mathrm{s}$ plastic transceiver module is proposed. The results show that the high SE of MWCNT composites exhibit 38-45 dB in the far-field source and $28-40 \mathrm{~dB}$ in the near-field source at a frequency range of $1-3 \mathrm{GHz}$, and over $14 \mathrm{~dB}$ for optical transceiver modules at a frequency of $2.5 \mathrm{GHz}$. Further research may develop the lower cost, lighter weight, and higher shielding optical transceiver module package employing MWCNT composites that have excellent electronic and mechanical properties when compared to conventional plastic composites. The paper is organized as follows. Section II describes the fabrication of plastic composites and plastic optical transceiver modules with transmission rate of $2.5 \mathrm{~Gb} / \mathrm{s}$. The measurement results of SE for plastic composites in the far-field source, realistic package in the near-field source of the monopole type, and the plastic optical transceiver module in the near-field source are presented in Section III. Conclusion and discussion are presented in Section IV.

\section{FABRICATION OF MWCNT COMPOSITES AND TRANSCEIVER MODULES}

\section{A. Material Properties of MWCNTs}

CNTs have excellent characteristics in both mechanism and electricity, such as a high yield strength, a high current density, a high conductivity, and no heat dissipation [12]. Basically, CNT is a hollow tube, which is constructed from carbon atoms. This kind of hollow structure of carbons is called fullerene. Usually, fullerenes consist of hexagons and pentagons that form a spherical shape. The most famous one is $\mathrm{C}_{60}$, which is built by 60 carbon atoms [12].

CNT is one of the fullerenes, but it is in a long hollow tube structure and its ends are semispherical-shaped cups. The tube wall atoms are hexagonal bonded like graphite and the tube-tip atoms are mixed with hexagonal- and pentagonal-bonded-like $\mathrm{C}_{60}$ [12]. Basically, it explains the origin of the high strength and excellent electrical characteristics. CNT has a high mechanical strength and a large aspect ratio. The latter aspect makes it suitable as a filler of plastic composite for conductive networks, which contribute to an increase in the conductivity of the composite for EM-shielding purpose.

MWCNT means a tube with many CNTs inside. These CNTs include metallic and semiconducting properties. And the CNTs inside MWCNT could be concentric or spirally spread. A MWCNT with both metallic and semiconducting CNTs inside is also metallic conductive [15], which can be used as a conductive filler to make plastic composites.

In this paper, the MWCNT is produced by arc-discharge deposit method (ADM). The electrodes are graphite rods and with a distance about $1-2 \mathrm{~mm}$. The applied voltage is $15-30 \mathrm{~V}$, the operated dc current is 50-150 A, and the applied argon gas pressure is $500-760$ bar. The deposit at cathode includes $25 \%$ MWCNT, 10\% carbon nanocapsules, and 65\% amorphous car-

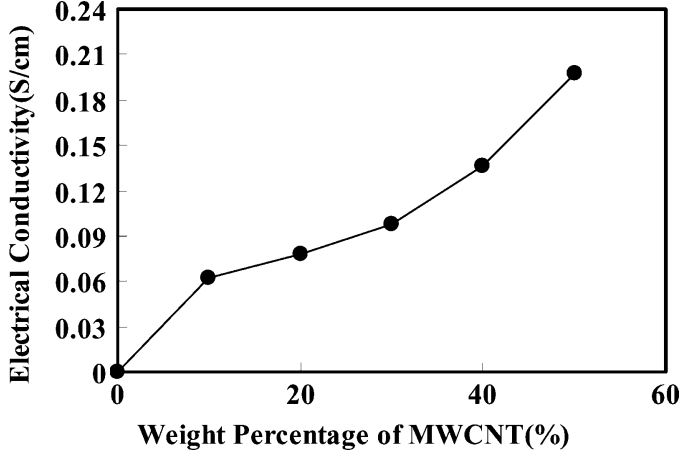

Fig. 1. Electrical conductivity as a function of mass fraction of MWCNT-LCP composite.

bons. The aspect ratio of these MWCNT is about 200-500 under SEM observation. The MWCNTs produced by ADM are not like those produced by chemical vaporization deposit method (CVD) where metal-catalyst particles existed. The metal-catalyst particles such as Fe and Co will influence the conductivity, which has been reported by Kim's group [16], [17].

\section{B. Material Properties of Multiwall Nanotube-LCP Composites}

The polymeric materials applied in this paper are LCPs [9]. The LCPs exhibit a highly-ordered structure in both the melt and solid states and are often applied to replace materials such as ceramics, metals, composites, and other plastics, because of their outstanding strength at high temperature and their resistance to chemicals, weathering, radiation, and flame. The LCPs have been applied for many injection and compression-molded parts for their excellent properties [3]-[5], [9]. All materials applied in this paper are in powder form for easy mixing and dispersion.

A compression-molding machine with temperature controllers was employed to mold specimens. The processing temperatures were set at $300{ }^{\circ} \mathrm{C}-350^{\circ} \mathrm{C}$ for $\mathrm{LCP}$ carbon-materialsfilled composites [9]. A compression-molded circular specimen with a diameter of $133 \mathrm{~mm}$ and a thickness of $1 \mathrm{~mm}$ was made as the EMI specimen. An annular $32 \mathrm{~mm}$ and $76 \mathrm{~mm}$ in inner and outer diameter, respectively, were cut for measuring the EM SE of the composites.

The conductivity of the MWCNT-LCP composite specimen was measured by a four-terminal technique. Fig. 1 shows the relation between the mass fraction of MWCNT and electrical conductivity. Fig. 1 revealed that the higher mass fraction of MWCNT led to a higher electrical conductivity. According to the basic EM-shielding theory, the higher conductivity is the higher shielding effectiveness. The more MWCNT is added, the more the overlapping conductive MWCNT networking, the higher the conductivity, and hence the higher the SE. Using SEM to examine the MWCNT-LCP composite, the morphology shows many trunk-like long fibers. However, in the MWCNTLCP composite, it is hard to distinguish MWCNT from LCP, since the LCP itself has a highly ordered structure and envelops the MWCNTs, as shown in Fig. 2(a). For the purpose 


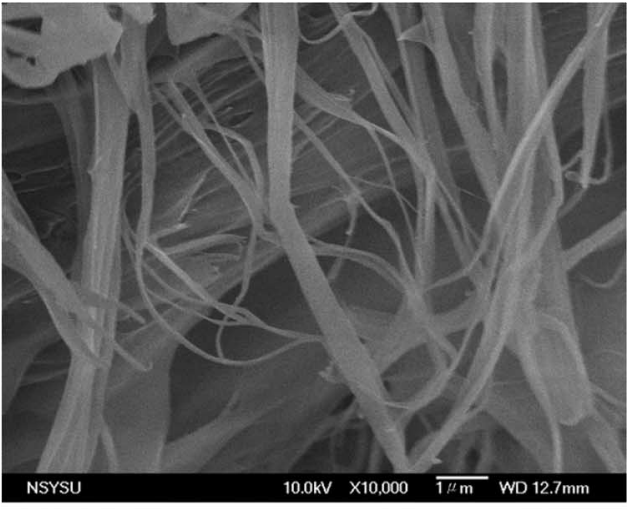

(a)

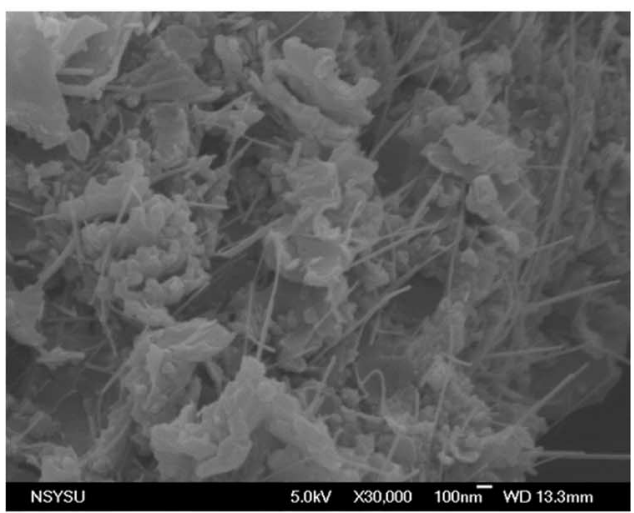

(b)

Fig. 2. (a) SEM photograph of MWCNTs embedded in LCPs. (b) SEM photograph of MWCNTs distribution in composite (locally).

of examining the real MWCNT dispersion, we use an ion technique to break the atomic bonds of LCP and MWCNT. Hence, the MWCNT morphology can be clearly observed, as shown in Fig. 2(b).

Tensile strength of the specimen exhibits the mechanical strength. Fig. 3 shows the relationship between the tensile strength and the mass fraction of MWCNT. The tensile strength increases as MWCNT mass fraction increases, since the role of MWCNT acts as enhancement of the composite. Fillers such as MWCNT with a high aspect ratio usually exhibit a high tensile strength and a good flexural strength.

\section{Optical Transceiver Modules}

An optical transceiver module consists of a $1.3-\mu \mathrm{m}$ laser (TOSA), a photodiode (ROSA), two ICs, and a plastic housing [3], [4]. These high-speed electric signals with a fast rising/falling edge would result in significant EMI problems as the transceiver modules are mounted onto the digital communication systems. Metallic housings are the general solution to reduce the EMI of the module for complying with the Federal Communications Commission (FCC) EMI regulation. But due to low cost and lightweight considerations, the plastic packaging or housing becomes one of the major trends for future optical transceiver module designs [1]-[5].

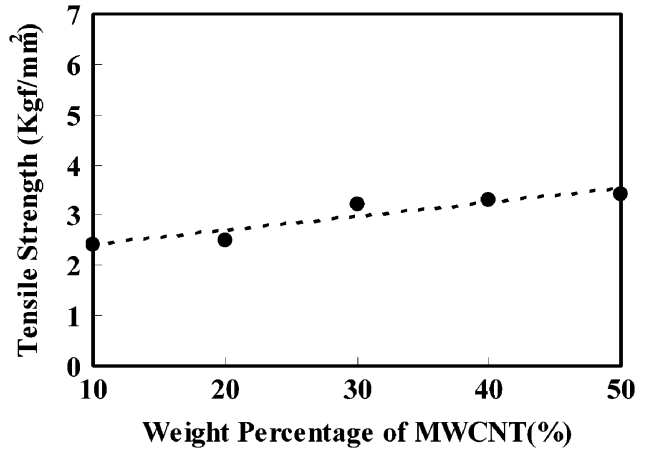

Fig. 3. Tensile strength as a function of mass fraction of MWCNT-LCP composite.

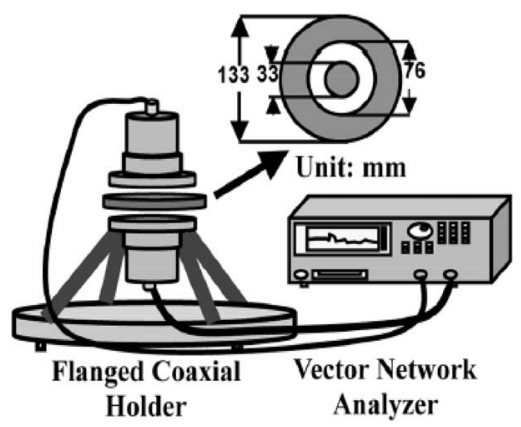

Fig. 4. Measurement setup for the shielding effectiveness of the plastic composites based on the ASTM D4935 method, and a cross section of the coaxialtype transmission-line holder.

The optical transceiver modules were fabricated by the MWCNT-LCP composites. The shape of the moldedpackage housing was a rectangular box with dimensions of $70 \mathrm{~mm} \times 30 \mathrm{~mm} \times 20 \mathrm{~mm}$. The weight percentage of MWCNT was $50 \%$. In general, the SE increases as the mass fraction of MWCNT increases. However, when the mass fraction of MWCNT increases more than 50\%, the MWCNT-LCP composite becomes easy to break when compressing the specimen. This might be due to less LCP to fill out all the space inside the composite specimen, which makes gaps or caves and reduces the strength of the specimen.

\section{Shielding Performance of MWCNT COMPosite AND TRANSCEIVER MODULE}

\section{A. Shielding Measurements of Plastic Composites in the Far-Field Source}

Fig. 4 shows a setup for the SE measurement and a cross section of the coaxial-type transmission lines holder. A flanged coaxial transmission-line holder was designed by following the ASTM D4935 method [18], which is used to measure the SE of a disk-shaped MWCNT-LCP specimen in the far-field source. The testing frequency range is from 1 to $3 \mathrm{GHz}$, since we focus on $2.5-\mathrm{Gb} / \mathrm{s}$ lightwave transmission applications. The diameter of the inner conductor was $33 \mathrm{~mm}$, and the outer conductor had inner and outer diameters of 76 and $133 \mathrm{~mm}$, respectively, according to the definition of ASTM D4935 [18]. The EM SE 


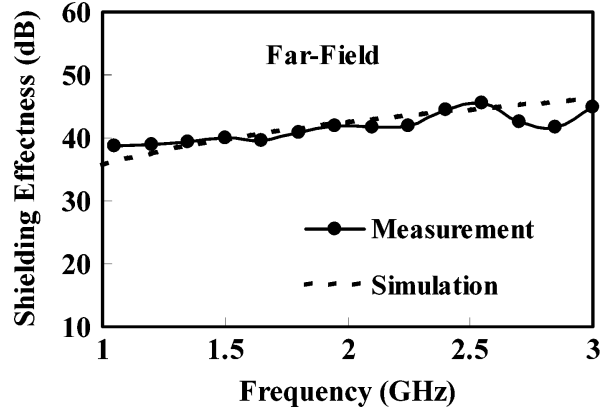

Fig. 5. Far-field measurement of 50\% weight percentage MWCNT-LCP composite.

of the MWCNT-LCP composites was measured by an insertion of the disk-shaped specimen with a diameter of $133 \mathrm{~mm}$ and a thickness of $1 \mathrm{~mm}$ between the two identical flanges. The purpose of the SE-test procedure is to quantitatively measure the insertion loss that results from introducing the test specimens. The result of the far-field measurement is shown in Fig. 5. The SE of the MWCNT-LCP composites was measured from 38 to $45 \mathrm{~dB}$ in the frequency range of $1-3 \mathrm{GHz}$, which is suitable for industry use. The SE of the MWCNT-LCP composite is also comparable to the other shielding plastic composites such as carbon fiber (CF)-nylon, CF-LCP, and WCCF-epoxy [4].

For a coaxial holder transmission-line circuit, there are significant parasitics between the holders and the specimen. The equivalent circuits of flanged coaxial holder with specimen can be modeled for theoretical calculation when the parasitic effects are considered [3], [8], [9], [19]. The calculated result of SE versus frequency are also shown in Fig. 5. Both measured (solid line) and calculated (dashed lines) results of the far field are in good correlation.

\section{B. Shielding Measurements of Realistic Package in the Near- Field Source of Monopole}

Near-field measurement is used to examine the SE of the specimen assembly, which is closer to the radiant source. It is more like the actual situation of a real application. A monopoletype antenna was used as the radiant source. Then, we put the monopole-type antenna into a built module box of the MWCNT-LCP composite to measure the difference of reference level and shielding level, which is the SE of the module box in the near-field situation. The SE measurement in a near-field source was carried out in a fully anechoic EM compatibility (EMC) chamber, as shown in Fig. 6 [3]-[5]. Because the hybrid absorbers combining the ferrite tiles and foam absorbers were aligned on the metal-shielded wall inside the chamber, good wave-absorbing performance could be achieved from $30 \mathrm{MHz}$ to $18 \mathrm{GHz}$ for the EMC chamber. The radiation source was put on a wooden table from which an antenna was seated at a distance of $3 \mathrm{~m}$ for receiving the radiated field. Because the interconnecting length on the optical transceiver module was generally about $1-2 \mathrm{~cm}$, an electric monopole with a 2 -cm length was used to emulate the radiation energy inside the molded housing.

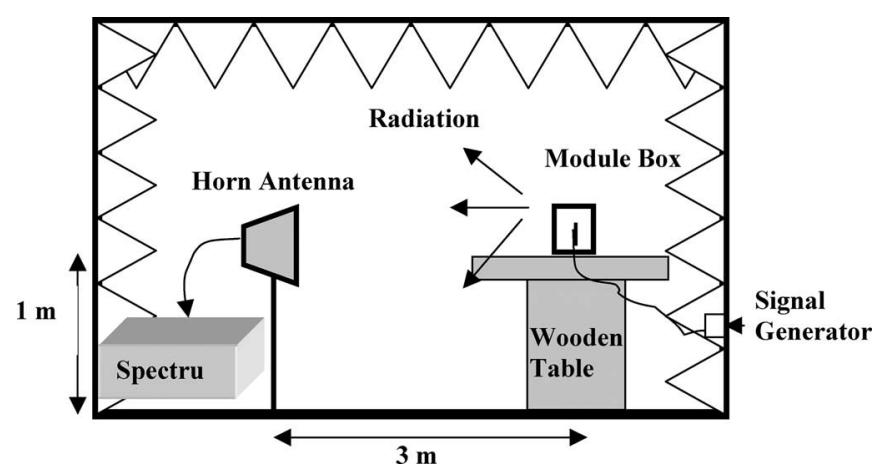

Fig. 6. SE-measurement setup for near-field monopole-type source in a fully anechoic EMC chamber.

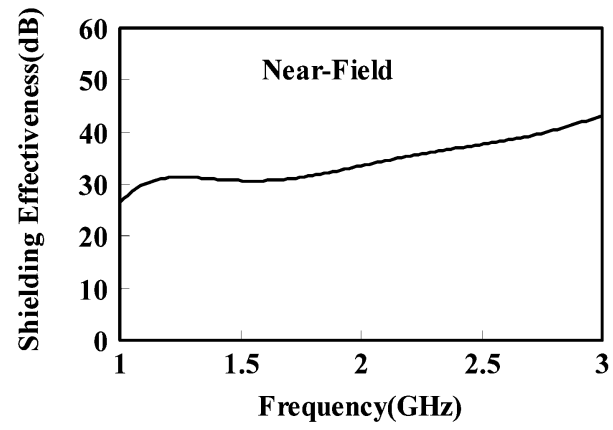

(a)

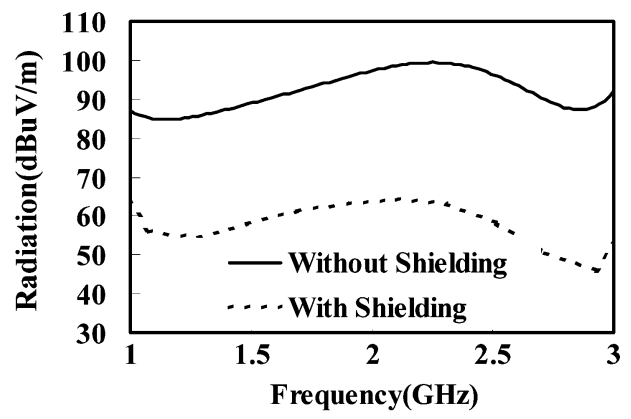

(b)

Fig. 7. (a) Near-field shielding effectiveness results and (b) received radiations of monotype antenna enclosed in a 50\% weight percentage MWCNT-LCP box.

Fig. 7 shows SE behavior versus frequency of the realistic housing fabricated by the MWCNT-LCP composites for a near-field radiation source of the monopole type. The SE of the MWCNT-LCP composites was measured from 28 to $40 \mathrm{~dB}$ in the frequency range of $1-3 \mathrm{GHz}$, as shown in Fig. 7(a). Fig. 7(b) shows the received radiation under the situations of with and without shielding boxes. The radiation of with and without shielding boxes showed 58 and $92 \mathrm{~dB} \cdot \mu \mathrm{V} / \mathrm{m}$ on average, respectively. Both values were far from the background white-noise level, the background white-noise level was around $30 \mathrm{~dB} \cdot \mu \mathrm{V} / \mathrm{m}$.

\section{Shielding Measurements of Packaged Transceiver Module in the Near-Field Source}

Optical transceiver module with transmission rate of $2.5 \mathrm{~Gb} / \mathrm{s}$ is tested to evaluate the EM shielding against emitted radiation 


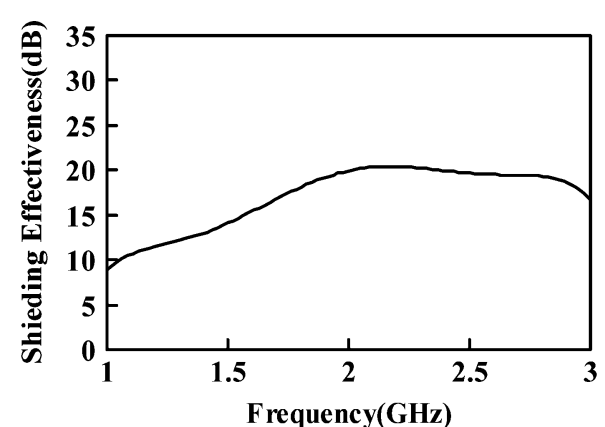

(a)

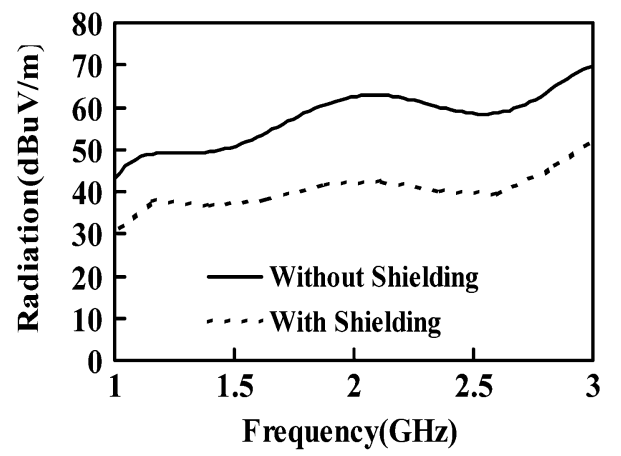

(b)

Fig. 8. (a) Near-field shielding effectiveness results and (b) received radiations of $2.5-\mathrm{Gb} / \mathrm{s}$ transmitter.

from the plastic packaging. The packaged transceiver was communicated with a golden specimen module (GSM) with identical functions to the module under test (MUT) [3]-[5]. A pattern generator (Tektronix GTS1250) transmitted the differential PRBS patterns $(2.5 \mathrm{~Gb} / \mathrm{s})$ to the GSM. Through a fiberoptic link, the data were received by the MUT working in the loopback mode inside the chamber.

An optical transceiver module usually consists of a transmitter and a receiver. In this paper, due to the limited housing space in the MWCNT-LCP composite box, the SE measurement of the optical transceiver module is carried out by separate transmitter and receiver-module measurements. The result of the near-field measurement of the $2.5-\mathrm{Gb} / \mathrm{s}$ transmitter module is shown in Fig. 8(a). The SE was about $20 \mathrm{~dB}$ on average in the 2.5-GHz region. Fig. 8(b) shows the received radiation under the situations of with and without shielding boxes. The received radiation of with and without shielding boxes were 40 and $60 \mathrm{~dB} \cdot \mu \mathrm{V} / \mathrm{m}$, on average, at a frequency of $2.5 \mathrm{GHz}$, respectively. And the radiation of with and without shielding boxes were 40 and $57 \mathrm{~dB} \cdot \mu \mathrm{V} / \mathrm{m}$ at the frequency range from 1 to $3 \mathrm{GHz}$, respectively. The radiation of $40 \mathrm{~dB} \cdot \mu \mathrm{V} / \mathrm{m}$ is closer to the background level, especially at lower frequencies.

Fig. 9(a) shows the results of the near-field measurement of the $2.5-\mathrm{Gb} / \mathrm{s}$ receiver module. The SE was about $14 \mathrm{~dB}$, on average, in the 2.5-GHz region. Fig. 9(b) shows the received radiation under the situations of with and without shielding boxes. The received radiation of with and without shielding boxes were 40 and $54 \mathrm{~dB} \cdot \mu \mathrm{V} / \mathrm{m}$, on average, at a frequency of $2.5 \mathrm{GHz}$, and the radiation of with and

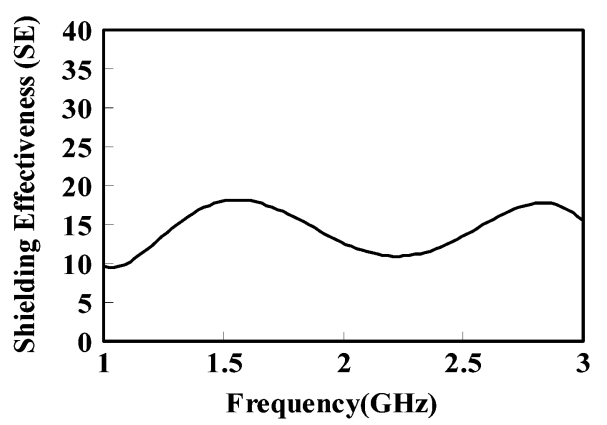

(a)

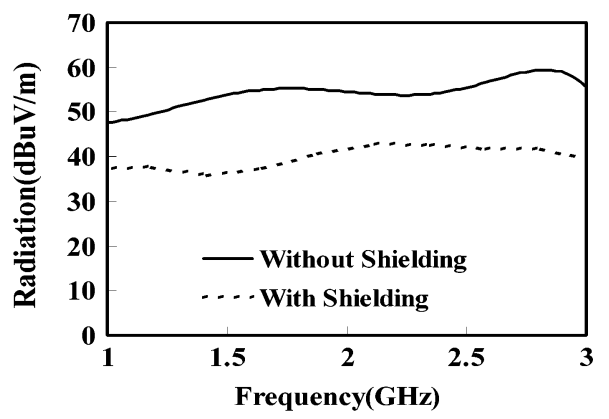

(b)

Fig. 9. (b) Near-field shielding effectiveness results and (b) received radiations of $2.5-\mathrm{Gb} / \mathrm{s}$ receiver.

without shielding boxes was 40 and $54 \mathrm{~dB} \cdot \mu \mathrm{V} / \mathrm{m}$, on average, in the frequency range $1-3 \mathrm{GHz}$. The radiation of $40 \mathrm{~dB} \cdot \mu \mathrm{V} / \mathrm{m}$ is closer to the background level, especially at lower frequencies.

The received radiation of optical transceiver without shielding box was measured as $59 \mathrm{~dB} \cdot \mu \mathrm{V} / \mathrm{m}$, on average, at the frequency range from 1 to $3 \mathrm{GHz}$ and $67 \mathrm{~dB} \cdot \mu \mathrm{V} / \mathrm{m}$, on average, in the $2.5-\mathrm{GHz}$ region. Based on the SE measurements of the transmitter and receiver modules from Figs. 8 and 9, the result of SE measurement for optical transceiver module at a frequency of $2.5 \mathrm{GHz}$ was to be $14 \mathrm{~dB}$ by using the worst case SE value from the receiver module. However, the expected radiation of the optical transceiver module was $53 \mathrm{~dB} \cdot \mu \mathrm{V} / \mathrm{m}(67$ $\mathrm{dB} \cdot \mu \mathrm{V} / \mathrm{m}-14 \mathrm{~dB}$ ), which is lower than the FCC regulation of $54 \mathrm{~dB} \cdot \mu \mathrm{V} / \mathrm{m}$ [20]. It is also suitable for industry use.

\section{DISCUSSION AND CONCLUSION}

MWCNT-LCP composite used as a novel EM-shielding material for packaging a plastic optical transceiver module was examined in both the near-field and the far-field sources. The results showed that the SE of MWCNT composites exhibited $38-45 \mathrm{~dB}$ in the far-field source and $28-40 \mathrm{~dB}$ in the near-field source at a frequency range of $1-3 \mathrm{GHz}$, and with an average of $14 \mathrm{~dB}$ for packaged optical transceiver modules at a frequency of $2.5 \mathrm{GHz}$. The SE behavior of the monotype antenna enclosed in the MWCNT-LCP composite box (Fig. 7) was higher than that of the optoelectronic devices (Fig. 8 for transmitter and Fig. 9 for receiver) in the $1-3-\mathrm{GHz}$ frequency range. This is due to the radiation of optoelectronic devices, which is not as high as the monotype antenna (about $92 \mathrm{~dB} \cdot \mu \mathrm{V} / \mathrm{m}$ ). Usually, a 
good design of EM-compatible (EMC) electrical circuit emits less radiation power. The radiation of the adopted optoelectronics devices (transmitter about $57 \mathrm{~dB} \cdot \mu \mathrm{V} / \mathrm{m}$, receiver about $54 \mathrm{~dB} \cdot \mu \mathrm{V} / \mathrm{m}$, and transceiver about $59 \mathrm{~dB} \cdot \mu \mathrm{V} / \mathrm{m}$ ) was not so high and not so far from the background white-noise level (around $30 \mathrm{~dB} \cdot \mu \mathrm{V} / \mathrm{m}$ ), especially at lower frequencies. This makes the margin against the background white noise small. Once the margin against the background white noise is too small, it shows a limited SE; however, the actual shielding ability of the module box is higher than that.

The results of receivers enclosed in module boxes also showed a low SE (Fig. 9) compared to that of the monotype antenna (Fig. 7). The adopted optoelectronic devices under test did not radiate as high as the monotype antenna. So, the results of monotype antenna enclosed in the module box might reflect the real shielding ability of the module box in the near-field situation. In brief, the module box of MWCNT-LCP composites have showed a good shielding ability to reduce the radiation from optoelectronic devices such as, transmitters, receivers, and transceivers under the testing frequency ranges of $1-3 \mathrm{GHz}$, and all the results meet FCC regulations [20].

In summary, a novel polymer-based MWCNT with a high SE for use in packaging a $2.5-\mathrm{Gb} / \mathrm{s}$ plastic transceiver module has been developed and fabricated. The MWCNT composites with their high SE are suitable for packaging low-cost and low-EMI optical transceiver modules used in Gigabit Ethernet or FTTH lightwave transmission systems.

\section{REFERENCES}

[1] K. Tatsuno, K. Yoshida, T. Kato, T. Hirataka, T. Miura, K. Fukuda, T. Ishikawa, M. Shimaoka, and T. Ishii, "High-performance and low cost plastic optical modules for access network system applications," J. Lightw. Technol., vol. 17, no. 7, pp. 1211-1216, Jul. 1999.

[2] M. Fukuda, F. Ichikawa, Y. Shuto, H. Sato, Y. Yamada, K. Kato, S. Tohno, H. Toba, T. Sugie, J. Yoshida, K. Suzuki, O. Suzuji, and S. Kondo, "Plastic module of laser diode and photodiode mounted on planar lightwave circuit for access network," J. Lightw. Technol., vol. 17, no. 9, pp. 1585-1592, Sep. 1999

[3] T.-L. Wu, W.-S. Jou, S. G. Dai, and W.-H. Cheng, "Effective electromagnetic shielding of plastic packaging in low-cost optical transceiver modules," J. Lightw. Technol., vol. 21, no. 6, pp. 1536-1543, Jun. 2003.

[4] W. H. Cheng, W. C. Hung, C. H. Lee, G. L. Hwang, W. S. Jou, and T. L. Wu, "Low-cost and low-electromagnetic-interference packaging of optical transceiver modules," J. Lightw. Technol., vol. 22, no. 9, pp. $2177-$ 2183, Sep. 2004.

[5] T. L Wu, M. C. Lin, C. W. Lin, T. T. Shih, and W. H. Cheng, "High electromagnetic susceptibility plastic package for $10 \mathrm{Gbit} / \mathrm{s}$ optical transceiver module," Electron. Lett., vol. 41, no. 8, pp. 494-495, Apr. 2005.

[6] P. B. Jana, A. K. Mallick, and S. K. De, "Effects of sample thickness and fiber aspect ratio on EMI shielding effectiveness of carbon fiber filled polychloroprene composites in the X-band frequency range," IEEE Trans. Electromagn. Compat., vol. 34, no. 4, pp. 478-481, Nov. 1992.

[7] P. F. Wilson, M. T. Ma, and J. W. Adams, "Techniques for measuring the electromagnetic shielding effectiveness of materials. I. Far-field source simulation," IEEE Trans. Electromagn. Compat., vol. 30, no. 3, pp. 239250, Aug. 1988.

[8] W. S. Jou, T. L. Wu, S. K. Chiu, and W. H. Cheng, "Electromagnetic shielding of nylon-66 composites applied to laser modules," J. Electron. Mater, vol. 30, no. 10, pp. 1287-1293, Oct. 2001.

[9] _ - "The influence of fiber orientation on electromagnetic shielding in liquid crystal polymers," J. Electron. Mater., vol. 31, no. 3, pp. 178-184, Mar. 2002.

[10] T.-L. Wu, W.-S. Jou, W.-C. Hung, C.-H. Lee, C.-W. Lin, and W.-H. Cheng, "High electromagnetic shielding of plastic package for 2.5 Gbps optical transceiver modules," IEEE Trans. Adv. Packag., vol. 28, no. 1, pp. 89-95, Feb. 2005.

[11] E. D. Minot, Y. Yaish, V. Sazonova, J. Y. Park, M. Brink, and P. L. McEuen, "Turning carbon nanotube band gaps with strain," Phys. Rev. Lett., vol.90, no. 15, pp. 154601-154604, Apr. 2003.

[12] K. B. K. Teo, R. G. Lacerda, M. H. Yang, A. S. Teh, L. A. W. Robinson, S. H. Dalal, N. L. Rupesinghe, M. Chhowalla, S. B. Lee, D. A. Jefferson, D. G. Hasko, G. A. J. Amaratunga, W. I. Milne, P. Legagneux, L. Gangloff, E. Minoux, J. P. Schnell, and D. Pribat, "Carbon nanotube technology for solid state and vacuum electronics," Proc. IEE Circuits Devices Syst., vol. 151 , no. 5, pp. 443-451, Oct. 2004.

[13] J. Li, Q. Ye, A. Cassell, H. T. Ng, R. Stevens, J. Han, and M. Meyyappan, "Bottom-up approach for carbon nanotube interconnects," Appl. Phys. Lett., vol. 82, no. 15, pp. 2491-2493, Apr. 2003.

[14] J. A. Misewich, R. Martel, P. Avouris, J. C. Tsang, S. Heinze, and J. Tersoff, "Electrically induced optical emission from a carbon nanotube FET," Science, vol. 300, pp. 783-786, May 2003.

[15] P. G. Collins and P. Avouris, "Multishell conduction in multiwalled carbon nanotubes," Appl. Phys. A, vol. 74, pp. 329-332, 2002.

[16] H. M. Kim, K. Kim, C. Y. Lee, J. Jooa, S. J. Cho, H. S. Yoon, D. A. Pejakovic, J. W. Yoo, and A. J. Epstein, "Electrical conductivity and electromagnetic interference shielding of multiwalled carbon nanotube composites containing Fe catalyst," Appl. Phys. Lett., vol. 84, no. 4, p. 26, Jan. 2004.

[17] H. M. Kim, K. Kim, S. J. Lee, J. Joo, H. S. Yoon, S. J. Cho, S. C. Lyu, and C. J. Lee, "Charge transport properties of composites of multiwalled carbon nanotube with metal catalyst and polymer: Application to electromagnetic interference shielding," Current Appl. Phys., vol. 4, pp. 577-580, 2004.

[18] "Standard testing method for measuring the electromagnet electromagnetic shielding effectiveness of planar materials," ASTM, Philadelphia, PA, Rep. ASTM D4935-92, 1987.

[19] P. F. Wilson, M. T. Ma, and J. W. Adams, "Techniques for measuring the electromagnetic shielding effectiveness of materials-Part I: Far-field source simulation," IEEE Trans. Electromagn. Compat., vol. 30, no. 3, pp. 239-250, Aug. 1988.

[20] C. R. Paul, Introduction to Electromagnetic Compatibility. New York: Wiley-Interscience, 1992, ch. 2, pp. 42-77.

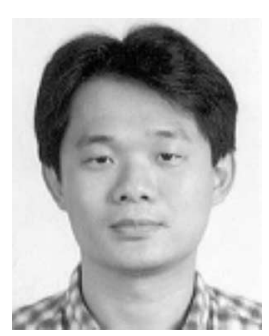

Chia-Ming Chang received the B.S. degree in electrical engineering from Tatung University, Taipei, Taiwan, R.O.C., in 1991, and the M.S. degree in electro-optical engineering from National Sun Yatsen University, Kaohsiung, Taiwan, in 2001. He is working toward the Ph.D. degree at National Sun Yat-sen University.

Currently, he is a Test Engineer with Philips Semiconductor Kaohsiung, Kaohsiung, Taiwan. His current research interests include optoelectronic packaging for telecommunication applications.

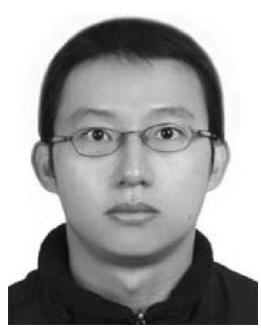

Jin-Chen Chiu received the B.S. degree in electrical engineering in 2004 from National Sun Yat-sen University, Kaohsiung, Taiwan, R.O.C., where he is currently working toward the M.S. and Ph.D. degrees.

His current research interests include optoelectronic packaging for telecommunication applications. 


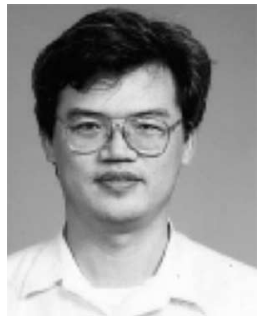

Wern-Shiang Jou was born in Taipei, Taiwan, R.O.C., on April 4, 1960. He received the Ph.D. degree in chemical engineering from the University of Maryland, College Park, in 1990.

From 1990 to 1992, he was a Research Associate in the Chemical Engineering Department of the University of Maryland and Cornell Injection Molding Program of the Cornell University, Ithaca, NY. He served as a Consultant for Suiyo Industry Company, Taiwan, R.O.C. Currently, he is an Associate Professor in the Department of Mold and Die Engineering, National Kaohsiung University of Applied Sciences, Kaoshiung, Taiwan. His research and development activities have been focused on the design and fabrication of new plastic composites, characterization of plastics, polymer processing, injection molding, and injection molded products. His current research interests include electromagnetic shielding materials and nanocomposites.

Dr. Jou is a member of the Society of Plastic Engineering and serves as a Secretary for the Association of Mold Technology Development, R.O.C.

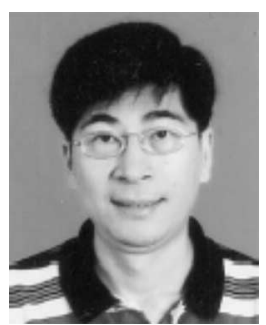

Tzong-Lin Wu (S'93-M'98-SM'04) was born in Hsinchu,Taiwan, R.O.C., on June 23, 1969. He received the B.S.E.E. and Ph.D. degrees from National Taiwan University, Taipei, Taiwan, in 1991 and 1995, respectively.

From 1995 to 1996, he was a Senior Engineer with Microelectronics Technology, Inc., Hsinchu, a wireless/satellite communication company, dedicated to the development of radio-frequency modules. From 1996 to 1998 , he was with the Central Research Institute, Tatung Company, Taipei, where he was involved with analysis and measurement of electromagnetic compatibility (EMC) problems of high-speed digital systems. In August 1998, he joined the faculty of the Electrical Engineering Department of National Sun Yat-sen University (NSYSU), Kaohsiung, Taiwan, as an Associate Professor. Currently, he is a Professor in the Department of Electrical Engineering, National Taiwan University. His currrent research interests include design and analysis of fiber-optic components and EMC/electromagnetic interference design for high-speed electronic and optical systems.

Dr. Wu received the Excellent Research Award from NSYSU in 2000 and the Outstanding Young Engineer Award from the Chinese Institute of Electrical Engineers in 2002. He was also listed in Who's Who in the World in 2001.

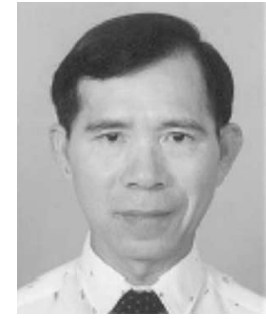

Wood-Hi Cheng (M'95-A'96-SM'00) was born in Changhua, Taiwan, R.O.C., on June 3, 1944. He received the Ph.D. degree in physics from Oklahoma State University, Stillwater, in 1978.

From 1978 to 1980, he was a Research Associate with Telecommunication Laboratories, Taiwan. From 1980 to 1984 , he was a Research Engineer with General Optronics, Edison, NJ. From 1984 to 1991, he was a Principal Design Engineer with Rockwell International, Newbury Park, CA. From 1991 to 1994 he was an Optoelectronic Packaging Manager with Tacan Corporation, Carlsbad, CA. He served as a Consultant for Chunghwa Telecom Laboratories, Opto-Electronics and System Laboratories, and ChungShan Institute of Science and Technology, all in Taiwan. Currently, he is a Professor with the Institute of Electro-Optical Engineering and Director of the Southern Taiwan Opto-Electronic Center of Excellence, National Sun Yat-sen University, Kaoshiung, Taiwan. His research and development activities have been focused on the design and fabrication of high-speed semiconductor lasers for lightwave communications, highly efficient light coupling from lasers into fibers, fiber couplers, characterization of III-V semiconductor materials, and optoelectronic packaging. His current research interests include the design, fabrication, and finite-element-method analysis for laser module packaging, high-speed laser module packaging for digital lightwave systems, fabrication of high-density wavelength division multiplexing components, and novel materials for electromagnetic shielding.

Dr. Cheng is a member of the Optical Society of America (OSA) and the Photonics Society of Chinese-Americans. He served as a Chair for the IEEE Lasers and Electro-Optics Society, Taipei Chapter, during 1999-2000, and serves as a Chair for the OSA, Taipei Chapter. 